\title{
Singular integral equations of convolution type with Hilbert kernel and a discrete jump problem
}

\section{Pingrun Li*}

\begin{abstract}
One class of singular integral equations of convolution type with Hilbert kernel is studied in the space $L^{2}[-\pi, \pi]$ in the article. Such equations can be changed into either a system of discrete equations or a discrete jump problem depending on some parameter via the discrete Laurent transform. We can thus solve the equations with an explicit representation of solutions under certain conditions.
\end{abstract}

MSC: 45E10; 45E05; 30E25

Keywords: singular integral equation; convolution type; Hilbert kernel; a discrete jump problem

\section{Introduction}

It is well known that singular integral equations and boundary value problems for analytic functions are the main branches of complex analysis and have a lot of applications, e.g., in elasticity theory, fluid dynamics, shell theory, underwater acoustics, and quantum mechanics. The theory is well developed by many authors [1-7]. Integral equations of convolution type are closely related to boundary value problem for an analytic function. There have been many papers studying integral equations with convolution type or singular type, see, for example, Litvinchuc [8], Li [9, 10], De-Bonis [11], Du [12], Jiang [13], among which a series of valuable achievements have been obtained. In recent years, the author [14] discussed some kinds of singular integral equations of convolution type with reflection and translation shifts. Subsequently, the author [15] studied one class of generalized boundary value problems for analytic functions and obtained the general solutions and the conditions of solvability.

The purpose of this article is to extend further the theory to a periodic singular integral equation of convolution type with Hilbert kernel. We remark that integral equations with periodicity have important applications in the elastic theory. Such equations can be changed into either a system of discrete equations or a discrete jump problem (that is, discrete boundary value problems) depending on some parameter via the discrete Laurent transform. We can thus solve the equations with an explicit representation of solutions in $L^{2}[-\pi, \pi]$ under certain conditions. This paper improves some results for the references [1-7].

(c) The Author(s) 2017. This article is distributed under the terms of the Creative Commons Attribution 4.0 International License (http://creativecommons.org/licenses/by/4.0/), which permits unrestricted use, distribution, and reproduction in any medium, provided you give appropriate credit to the original author(s) and the source, provide a link to the Creative Commons license, and indicate if changes were made. 
We shall consider the following singular integral equation of convolution type with Hilbert kernel and periodicity:

$$
\begin{aligned}
& a_{1} f+b_{1} H f+K_{1} * f+M_{1} * H f+\xi\left(a_{2} f+b_{2} H f+K_{2} * f+M_{2} * H f\right) \\
& \quad=\left(c_{1}+c_{2} \xi\right) G,
\end{aligned}
$$

where $K_{j}, M_{j}, G \in L^{2}[-\pi, \pi]$ are given functions, $a_{j}, b_{j}, c_{j} \in \mathbb{R}$ for any $j=1,2$, and $f \in$ $L^{2}[-\pi, \pi]$ is an unknown function. We denote by $*$ the convolution operator and by $H f$ the Hilbert type singular integral of $f$, that is,

$$
H f=\frac{1}{\pi} \int_{-\pi}^{\pi} f(\tau) \cot \frac{\tau-\theta}{2} d \tau
$$

Assumption A We shall make the following assumption:

(i) $a_{1}^{2}+a_{2}^{2} \neq 0, b_{1}^{2}+b_{2}^{2} \neq 0$.

(ii) There exist constants $a, b, c, d \in \mathbb{R}$ and some $m \in \mathbb{N}$ such that

$$
\xi(\theta)=\frac{a+b \tan \frac{m}{2} \theta}{c+d \tan \frac{m}{2} \theta}
$$

whenever $a d-b c \neq 0$; otherwise we have $\xi(\theta)=C$ (constant). We shall also represent $\xi(\theta)$ as

$$
\xi(\theta)=\frac{\alpha^{+}+\alpha^{-} e^{i m \theta}}{\beta^{+}+\beta^{-} e^{i m \theta}}
$$

where

$$
\alpha^{ \pm}=a \pm i b, \quad \beta^{ \pm}=c \pm i d
$$

Let $\mathbb{L}, \mathbb{L}^{-1}$ be the discrete Laurent transform and the inverse transform, respectively. We denote

$$
\begin{aligned}
& S^{ \pm}=\left(a_{2} a+a_{1} c\right) \pm i\left(a_{2} b+a_{1} d\right), \\
& T^{ \pm}=\left(b_{1} c+b_{2} a\right) \pm i\left(b_{1} d+b_{2} b\right), \\
& B_{k}^{(j)}=\mathbb{L}^{-1} K_{j}, \quad C_{k}^{(j)}=\mathbb{L}^{-1} M_{j}
\end{aligned}
$$

and

$$
w_{k}=\frac{\Delta_{k-m}^{-}}{\Delta_{k}^{+}},
$$

where

$$
\Delta_{k}^{ \pm}=S^{ \pm}+\beta^{ \pm} B_{k}^{(1)}+\alpha^{ \pm} B_{k}^{(2)}+2 i \operatorname{sgn} k\left(T^{ \pm}+\beta^{ \pm} C_{k}^{(1)}+\alpha^{ \pm} C_{k}^{(2)}\right) .
$$


Assumption B To assure the solvability of Eq. (1.1), we need to make the assumption

$$
\sum_{k \in \mathbb{Z}}\left(\log \frac{S^{+}}{S^{-}} w_{k}\right) e^{i k \theta_{j}}=0,
$$

where

$$
\theta_{j}=\frac{2 j}{m} \pi
$$

for any $j=0, \pm 1, \ldots, \pm\left[\frac{m}{2}\right]$. Via using the method of complex analysis, we can take a continuous branch of $\log \left(\frac{S^{+}}{S^{-}} w_{k}\right)$ such that $\left\{\log \left(\frac{S^{+}}{S^{-}} w_{k}\right)\right\}_{k \in \mathbb{Z}} \in l^{2}$.

There exist constants $\varepsilon_{0} \in(0,1)$ and $k_{0} \in \mathbb{N}$ such that when $|k|>k_{0}$ we have

$$
\left|\Delta_{k}^{+}\right|>\varepsilon_{0}, \quad\left|\Delta_{k-m}^{-}\right|>\varepsilon_{0}
$$

We can thus introduce the equalities

$$
\begin{aligned}
A_{k}= & \frac{1}{S^{-} \Delta_{k}^{+}}\left\{\beta^{-} S^{+} B_{k-m}^{(1)}-\beta^{+} S^{-} B_{k}^{(1)}+\alpha^{-} S^{+} B_{k-m}^{(2)}-\alpha^{+} S^{-} B_{k}^{(2)}\right. \\
& \left.+2 i\left[\left(\beta^{-} S^{+} C_{k-m}^{(1)}+\alpha^{-} S^{+} C_{k-m}^{(2)}\right) \operatorname{sgn}(k-m)-\left(\beta^{+} S^{-} C_{k}^{(1)}+\alpha^{+} S^{-} C_{k}^{(2)}\right) \operatorname{sgn} k\right]\right\} .
\end{aligned}
$$

We may make $\eta_{k}$ such that

$$
\eta_{k}=\left(1+A_{k}\right) \eta_{k-m}
$$

Assumption C To assure the solvability, we need also

$$
\sum_{k \in \mathbb{Z}} \frac{H_{k}}{\eta_{k}} e^{i k \theta_{j}^{\prime}}=0,
$$

where $\eta_{k}$ is determined by (1.4),

$$
H_{k}=\frac{1}{\Delta_{k}^{+}}\left(E^{+} g_{k}+E^{-} g_{k-m}\right), \quad\left\{g_{k}\right\}_{k \in \mathbb{Z}}=\mathbb{L}^{-1} G
$$

and

$$
E^{ \pm}=\left(c_{1} c+c_{2} a\right) \pm i\left(c_{2} b+c_{1} d\right)
$$

In order to illustrate that Eq. (1.1) has a solution, at the end of Section 3, we shall present an example and satisfy the above conditions (Assumptions A-C), then we can conclude that a solution set of (1.1) is not empty.

Now we can state our main result, and we will prove it in Section 3.

Theorem 1.1 Under Assumptions A-C, Eq. (1.1) has a solution in $L^{2}[-\pi, \pi]$. 
(i) When $\Delta_{k}^{+} \neq 0, \Delta_{k-m}^{-} \neq 0$ for any $k \in \mathbb{Z}$, the solution of Eq. (1.1) is given by

$$
f(\theta)=\sum_{k=-\infty}^{+\infty} f_{k} e^{i k \theta}
$$

and the coefficients $f_{k}$ are determined uniquely by the formula

$$
\Delta_{k}^{+} f_{k}+\Delta_{k-m}^{-} f_{k-m}=E^{+} g_{k}+E^{-} g_{k-m}
$$

(ii) When $\Delta_{n_{j}}^{+}=0, \Delta_{n_{j}-m}^{-}=0\left(\left|n_{j}\right| \leq k_{0} ; j=1,2, \ldots, p_{1}\right)$, then

$$
E^{+} g_{n_{j}}+E^{-} g_{n_{j}-m}=0
$$

must be satisfied, and $f_{n_{j}}$ can be taken to be an arbitrary constant $c_{n_{j}}\left(1 \leq j \leq p_{1}\right)$, then the solution of Eq. (1.1) is

$$
f(\theta)=\sum_{j=1}^{p_{1}} c_{n_{j}} e^{i n_{j} \theta}+\sum_{k=-\infty, k \neq n_{j}}^{+\infty} f_{k} e^{i k \theta} .
$$

(iii) When $\Delta_{n_{j}^{\prime}}^{+}=0, \Delta_{n_{j}^{\prime}-m}^{-} \neq 0\left(\left|n_{j}^{\prime}\right| \leq k_{0} ; j=1,2, \ldots, p_{2}\right), f_{n_{j}^{\prime}}$ are given by the following formula:

$$
f_{n_{j}^{\prime}}=\frac{1}{\Delta_{n_{j}^{\prime}}^{-}}\left(E^{+} g_{n_{j}^{\prime}+m}+E^{-} g_{n_{j}^{\prime}}\right),
$$

then the solution of Eq. (1.1) is

$$
f(\theta)=\sum_{j=1}^{p_{2}} f_{n_{j}^{\prime}} e^{i n_{j}^{\prime} \theta}+\sum_{k=-\infty, k \neq n_{j}^{\prime}}^{+\infty} f_{k} e^{i k \theta} .
$$

(iv) When $\Delta_{n_{j}^{\prime \prime}}^{+} \neq 0, \Delta_{n_{j}^{\prime \prime}-m}^{-}=0\left(\left|n_{j}^{\prime \prime}\right| \leq k_{0} ; j=1,2, \ldots, p_{3}\right), f_{n_{j}^{\prime \prime}}$ are given by the following formula:

$$
f_{n_{j}^{\prime \prime}}=\frac{1}{\Delta_{n_{j}^{\prime \prime}}^{+}}\left(E^{+} g_{n_{j}^{\prime \prime}}+E^{-} g_{n_{j}^{\prime \prime}-m}\right),
$$

then the solution of Eq. (1.1) is

$$
f(\theta)=\sum_{j=1}^{p_{3}} f_{n_{j}^{\prime \prime}} e^{i n_{j}^{\prime \prime} \theta}+\sum_{k=-\infty, k \neq n_{j}^{\prime \prime}}^{+\infty} f_{k} e^{i k \theta} .
$$

In (ii)-(iv), when $k \neq n_{1}, n_{2}, \ldots, n_{p_{1}}, n_{1}^{\prime}, n_{2}^{\prime}, \ldots, n_{p_{2}}^{\prime}, n_{1}^{\prime \prime}, n_{2}^{\prime \prime}, \ldots, n_{p_{3}}^{\prime \prime}$, the coefficients $f_{k}$ are determined uniquely by (1.6). 


\section{Hilbert transform and its discrete Laurent transform}

The crucial tool to study the Hilbert transform is to calculate its discrete Laurent transform.

For any $f \in L^{2}[-\pi, \pi]$, its Hilbert transform is defined as

$$
H f(\theta)=\frac{1}{\pi} \int_{-\pi}^{\pi} f(\tau) \cot \frac{\tau-\theta}{2} d \tau .
$$

Associated to the operator $H$ is the operator $\tilde{H}$ defined by

$$
\tilde{H} f(\theta)=\frac{1}{\pi} \int_{-\pi}^{\pi} f(\tau) \cot \frac{\tau+\theta}{2} d \tau,
$$

that is,

$$
\tilde{H} f(\theta)=H f(-\theta) .
$$

It is well known that the Hilbert transform $H$ as well $\tilde{H}$ is a self-map of the space $L^{2}[-\pi, \pi]$ in virtue of the Riesz theorem (see, e.g., [16]).

Let $l^{2}(\mathbb{Z})$ be a linear space consisting of sequences $\left\{f_{k}\right\}_{k \in \mathbb{Z}}$ for which

$$
\sum_{k=-\infty}^{+\infty}\left|f_{k}\right|^{2}<+\infty
$$

Definition 2.1 The discrete Laurent transform

$$
\mathbb{L}: l^{2}(\mathbb{Z}) \longrightarrow L^{2}[-\pi, \pi]
$$

is defined by

$$
\mathbb{L}\left\{f_{k}\right\}_{k \in \mathbb{Z}}=\sum_{k=-\infty}^{+\infty} f_{k} e^{i k \theta}=: f(\theta)
$$

for any $f=\left\{f_{k}\right\}_{k \in \mathbb{Z}} \in l^{2}(\mathbb{Z})$. Its inverse transform is clearly given by

$$
\mathbb{L}^{-1}[f]_{k}=\left\{f_{k}\right\}_{k \in \mathbb{Z}}
$$

with

$$
f_{k}=\frac{1}{2 \pi} \int_{-\pi}^{\pi} f(\theta) e^{-i k \theta} d \theta, \quad \forall k \in \mathbb{Z} .
$$

Now we come to calculate the inverse discrete Laurent transform of a function which is a Hilbert transform of a given function. The result shall be crucial to studying the singular integral equations.

Lemma 2.1 Let $f(\theta) \in L^{2}[-\pi, \pi]$ with its discrete Laurent transform

$$
\left\{f_{k}\right\}_{k \in \mathbb{Z}}=\mathbb{L}^{-1} f(\theta) .
$$


Then we have

$$
\begin{aligned}
& \mathbb{L}^{-1}(H f(\theta))=\left\{2 i f_{k} \operatorname{sgn} k\right\}_{k \in \mathbb{Z}}, \\
& \mathbb{L}^{-1}(\tilde{H} f(\theta))=\left\{-2 i f_{-k} \operatorname{sgn} k\right\}_{k \in \mathbb{Z}} .
\end{aligned}
$$

Proof Since

$$
\cot \frac{\tau-\theta}{2}=\frac{e^{i \tau}}{e^{i \tau}-e^{i \theta}}-\frac{e^{-i \tau}}{e^{-i \tau}-e^{-i \theta}}
$$

by Definition 2.1 we have

$$
H f(\theta)=\frac{i}{\pi} \int_{-\pi}^{\pi} f(\tau)\left(\frac{e^{i \tau}}{e^{i \tau}-e^{i \theta}}-\frac{e^{-i \tau}}{e^{-i \tau}-e^{-i \theta}}\right) d \tau .
$$

For any $f(\theta) \in L^{2}[-\pi, \pi]$, we already know that $H f(\theta) \in L^{2}[-\pi, \pi]$ so that $\mathbb{L}^{-1}(H f(\theta)) \in$ $l^{2}(\mathbb{Z})$. We denote

$$
\mathbb{L}^{-1}(H f(\theta))=\left\{g_{k}\right\}_{k \in \mathbb{Z}}
$$

Then

$$
\begin{aligned}
g_{k} & =\frac{1}{2 \pi} \int_{-\pi}^{\pi} H f(\theta) e^{-i k \theta} d \theta \\
& =\frac{i}{2 \pi^{2}} \int_{-\pi}^{\pi} \int_{-\pi}^{\pi} f(\tau) \frac{e^{i \tau} e^{-i k \theta}}{e^{i \tau}-e^{i \theta}} d \tau d \theta-\frac{i}{2 \pi^{2}} \int_{-\pi}^{\pi} \int_{-\pi}^{\pi} f(\tau) \frac{e^{-i \tau} e^{-i k \theta}}{e^{-i \tau}-e^{-i \theta}} d \tau d \theta \\
& =: I_{1}-I_{2} .
\end{aligned}
$$

For the first term, we have

$$
I_{1}=\frac{i}{2 \pi^{2}} \int_{-\pi}^{\pi}\left(\int_{-\pi}^{\pi} \frac{e^{-i k \theta}}{e^{i \tau}-e^{i \theta}} d \theta\right) f(\tau) e^{i \tau} d \tau .
$$

By a change of variable $t=\exp (i \theta)$, the inner integral above becomes

$$
M(\tau):=\int_{-\pi}^{\pi} \frac{e^{-i k \theta}}{e^{i \tau}-e^{i \theta}} d \theta=\int_{|t|=1} \frac{t^{-k}}{\left(e^{i \tau}-t\right) i t} d t
$$

Applying the extended residue theory, we thus obtain

$$
M(\tau)= \begin{cases}\frac{\pi}{e^{i(k+1) \tau}}, & k \geq 0 \\ -\frac{\pi}{e^{i(k+1) \tau}}, & k<0 .\end{cases}
$$

Therefore, when $k \geq 0$, we have

$$
I_{1}=\frac{i}{2 \pi^{2}} \int_{-\pi}^{\pi} \frac{\pi}{e^{i(k+1) \tau}} f(\tau) e^{i \tau} d \tau=\frac{i}{2 \pi} \int_{-\pi}^{\pi} f(\tau) e^{-i k \tau} d \tau=i f_{k}
$$


and similarly

$$
I_{1}=-i f_{k}, \quad k<0 .
$$

In other words,

$$
I_{1}=i f_{k} \operatorname{sgn} k, \quad \forall k \in \mathbb{Z}
$$

Similarly, we have

$$
I_{2}=-i f_{k} \operatorname{sgn} k, \quad \forall k \in \mathbb{Z}
$$

As a result,

$$
g_{k}=I_{1}-I_{2}=2 i f_{k} \operatorname{sgn} k, \quad \forall k \in \mathbb{Z}
$$

The other equality can be proven similarly.

Lemma 2.2 Let $f(\theta)=\sum_{k=-\infty}^{\infty} f_{k} e^{i k \theta}$ and $f \in l^{1}$, then $f(\theta) \in L^{2}[-\pi, \pi]$ if and only iff $\in l^{2}$.

Proof Since $f(\theta) \in L^{2}[-\pi, \pi]$ and $f \in l^{1}$, then $\sum_{k=-\infty}^{\infty} f_{k}$ is convergent absolutely and uniformly. It is easy to see that

$$
\begin{aligned}
\int_{-\pi}^{\pi}|f(\theta)|^{2} d \theta & =\int_{-\pi}^{\pi} f(\theta) \overline{f(\theta)} d \theta=\int_{-\pi}^{\pi} \sum f_{k} e^{i k \theta} \sum \bar{f}_{j} e^{-i j \theta} d \theta \\
& =\int_{-\pi}^{\pi} \sum_{k, j=-\infty}^{\infty} f_{k} \bar{f}_{j} e^{i(k-j) \theta} d \theta=2 \pi \sum_{k=-\infty}^{\infty}\left|f_{k}\right|^{2} .
\end{aligned}
$$

The proof of Lemma 2.2 is complete.

Finally, we remark that

$$
\mathbb{L}^{-1}[f * g(\theta)]=\left\{f_{k} g_{k}\right\}_{k \in \mathbb{Z}}
$$

for any $f, g \in L^{2}[-\pi, \pi]$ with the discrete Laurent transforms $\left\{f_{k}\right\}_{k \in \mathbb{Z}}$ and $\left\{g_{k}\right\}_{k \in \mathbb{Z}}$, respectively. Here the convolution in $L^{2}[-\pi, \pi]$ is defined by

$$
(f * g)(\theta)=\frac{1}{2 \pi} \int_{-\pi}^{\pi} f(\theta-\tau) g(\tau) d \tau .
$$

\section{Problem presentation and solution}

In this section, we study the method of solution for Eq. (1.1). By Euler's formula $e^{i m \theta}=$ $\cos m \theta+i \sin m \theta$, Eq. (1.1) can be written as

$$
\begin{aligned}
S^{+} f+ & T^{+} H f+\beta^{+} K_{1} * f+\alpha^{+} K_{2} * f+\beta^{+} M_{1} * H f+\alpha^{+} M_{2} * H f-E^{+} G \\
& +e^{i m \theta}\left(S^{-} f+T^{-} H f+\beta^{-} K_{1} * f+\alpha^{-} K_{2} * f+\beta^{-} M_{1} * H f+\alpha^{-} M_{2} * H f-E^{-} G\right) \\
= & 0 .
\end{aligned}
$$


In view of Lemma 2.1 , by applying $\mathbb{L}^{-1}$ to both sides of (3.1), we see that (3.1) is readily reduced to the equation

$$
\Delta_{k}^{+} f_{k}-E^{+} g_{k}+\Delta_{k-m}^{-} f_{k-m}-E^{-} g_{k-m}=0
$$

where

$$
\begin{aligned}
& \left\{f_{k}\right\}_{k \in \mathbb{Z}}=\mathbb{L}^{-1} f, \quad\left\{g_{k}\right\}_{k \in \mathbb{Z}}=\mathbb{L}^{-1} G, \\
& \left\{B_{k}^{(j)}\right\}_{k \in \mathbb{Z}}=\mathbb{L}^{-1} K_{j}, \quad\left\{C_{k}^{(j)}\right\}_{k \in \mathbb{Z}}=\mathbb{L}^{-1} M_{j}
\end{aligned}
$$

with any $j=1,2 ; k \in \mathbb{Z}$.

Since by assumption, for each $j=1,2$,

$$
\left\{B_{k}^{(j)}\right\}_{k \in \mathbb{Z}} \in l^{2}(\mathbb{Z}), \quad\left\{C_{k}^{(j)}\right\}_{k \in \mathbb{Z}} \in l^{2}(\mathbb{Z})
$$

it follows that

$$
\lim _{k \rightarrow \infty} B_{k}^{(j)}=0, \quad \lim _{k \rightarrow \infty} C_{k}^{(j)}=0 .
$$

Therefore,

$$
\lim _{k \rightarrow \infty} \Delta_{k}^{+}=S^{+} \pm 2 i T^{+} \neq 0, \quad \lim _{k \rightarrow \infty} \Delta_{k}^{-}=S^{-} \pm 2 i T^{-} \neq 0
$$

which means that for any $\varepsilon_{0}$ sufficiently small, there exists $k_{0}>0$ such that when $|k|>k_{0}$ we have

$$
\left|\Delta_{k}^{+}\right|>\varepsilon_{0}, \quad\left|\Delta_{k-m}^{-}\right|>\varepsilon_{0}
$$

Case 1: $|k|>k_{0}$.

Since $\left|\Delta_{k}^{+}\right|>\varepsilon_{0},\left|\Delta_{k-m}^{-}\right|>\varepsilon_{0}$ for $|k|>k_{0}$, it follows from (1.6) that

$$
f_{k}=-\frac{\Delta_{k-m}^{-}}{\Delta_{k}^{+}} f_{k-m}+\frac{1}{\Delta_{k}^{+}}\left(E^{+} g_{k}+E^{-} g_{k-m}\right), \quad \forall|k|>k_{0},
$$

so that Eq. (3.3) can be rewritten as

$$
f_{k}=-w_{k} f_{k-m}+H_{k}, \quad \forall|k|>k_{0}
$$

Denote

$$
\rho=\frac{S^{-}}{S^{+}}, \quad w_{k}=\rho\left(1+A_{k}\right)
$$

When $|k|$ is large enough, we see that $\operatorname{sgn}(k-m)$ and $\operatorname{sgn} k$ equal 1 or -1 simultaneously so that

$$
S^{+} T^{-} \operatorname{sgn}(k-m)-S^{-} T^{+} \operatorname{sgn} k=0 .
$$


Consequently,

$$
\begin{aligned}
A_{k}= & \frac{1}{S^{-} \Delta_{k}^{+}}\left\{\beta^{-} S^{+} B_{k-m}^{(1)}-\beta^{+} S^{-} B_{k}^{(1)}+\alpha^{-} S^{+} B_{k-m}^{(2)}-\alpha^{+} S^{-} B_{k}^{(2)}\right. \\
& \left.+2 i\left[\left(\beta^{-} S^{+} C_{k-m}^{(1)}+\alpha^{-} S^{+} C_{k-m}^{(2)}\right) \operatorname{sgn}(k-m)-\left(\beta^{+} S^{-} C_{k}^{(1)}+\alpha^{+} S^{-} C_{k}^{(2)}\right) \operatorname{sgn} k\right]\right\} .
\end{aligned}
$$

Therefore, (3.4) becomes a discrete jump problem

$$
f_{k}=-\rho\left(1+A_{k}\right) f_{k-m}+H_{k}, \quad \forall|k|>k_{0} .
$$

In order to solve (3.6), we may make $\eta_{k}$ such that

$$
\eta_{k}=\left(1+A_{k}\right) \eta_{k-m}
$$

with

$$
\varepsilon_{0}<\left|\eta_{k}\right|<\varepsilon_{0}^{-1} .
$$

First, we need to construct $\eta_{k}$. By taking logarithms on both sides of (3.7) and denoting

$$
M_{k}=\log \left(1+A_{k}\right), \quad o_{k}=\log \eta_{k}
$$

we get

$$
o_{k}=o_{k-m}+M_{k} \text {, }
$$

where we have taken a continuous branch of $\log \left(1+A_{k}\right)$ so that $\left\{\log \left(1+A_{k}\right)\right\}_{k \in \mathbb{Z}} \in l^{2}$. Taking the Laurent transform $\mathbb{L}$ on both sides of (3.8) yields

$$
O(\theta)=e^{i m \theta} O(\theta)+\tilde{M}(\theta)
$$

that is,

$$
\left(1-e^{i m \theta}\right) O(\theta)=\tilde{M}(\theta)
$$

where

$$
O(\theta)=\mathbb{L} o, \quad \tilde{M}(\theta)=\mathbb{L} M, \quad o=\left\{o_{k}\right\}_{k \in \mathbb{Z}}, \quad M=\left\{M_{k}\right\}_{k \in \mathbb{Z}} .
$$

Notice that when $\tilde{M}\left(\theta_{j}\right) \neq 0$, Eq. (3.8) is not solvable. This means $\tilde{M}\left(\theta_{j}\right)=0$ or, equivalently, Assumption B becomes the solvability conditions of Eq. (3.8), where $\theta_{j}$ are $1+\left[\frac{\mathrm{m}}{2}\right]$ roots on $[-\pi, \pi]$ for equation $1-e^{i m \theta}=0$.

Finally, from (3.9) we get

$$
\eta_{k}=\exp o_{k}, \quad o_{k}=\mathbb{L}^{-1} \frac{\tilde{M}(\theta)}{1-e^{i m \theta}} .
$$


Now we come to solve Eq. (3.6). Denote

$$
p_{k}=\frac{1}{\eta_{k}} f_{k}, \quad q_{k}=\frac{1}{\eta_{k}} H_{k}
$$

and rewrite (3.6) as

$$
p_{k}=-\rho p_{k-m}+q_{k}
$$

Due to $\left\{H_{k}\right\}_{k \in \mathbb{Z}} \in l^{2}(\mathbb{Z}),\left\{\eta_{k}\right\}_{k \in \mathbb{Z}} \in l^{2}(\mathbb{Z})$, we can know that $\left\{p_{k}\right\}_{k \in \mathbb{Z}} \in l^{2}(\mathbb{Z}),\left\{q_{k}\right\}_{k \in \mathbb{Z}} \in$ $l^{2}(\mathbb{Z})$. Taking the Laurent transform $\mathbb{L}$ on both sides of (3.11), we thus obtain

$$
P(\theta)=-\rho e^{i m \theta} P(\theta)+Q(\theta)
$$

where $P(\theta)=\mathbb{L} p$ and $Q(\theta)=\mathbb{L} q$ with $p=\left\{p_{k}\right\}_{k \in \mathbb{Z}}$ and $q=\left\{q_{k}\right\}_{k \in \mathbb{Z}}$.

Owing to $|\rho|=1$, we know that $1+\rho e^{i m \theta}$ has a finite number of zero points, say $\theta_{1}^{\prime}, \theta_{2}^{\prime}, \ldots, \theta_{n}^{\prime}$ in $[-\pi, \pi]$.

The same approach as in the discussion of Eq. (3.8) shows that (3.11) is not solvable if $Q\left(\theta_{j}^{\prime}\right) \neq 0$. Therefore, $Q\left(\theta_{j}^{\prime}\right)=0(j=1,2, \ldots, n)$, or equivalently, Assumption $C$ becomes the solvability conditions of Eq. (3.11).

Under Assumption C, (3.12) becomes

$$
P(\theta)=\frac{Q(\theta)}{1+\rho e^{i m \theta}} .
$$

This determines $p_{k}$ so does $f_{k}=p_{k} \eta_{k}$.

Case $2:|k| \leq k_{0}$.

We split the situation into four cases.

(a) $\Delta_{k}^{+}=0$ and $\Delta_{k-m}^{-}=0$ for some $|k| \leq k_{0}$. By (3.2), we have

$$
E^{+} g_{k}=-E^{-} g_{k-m}
$$

and $f_{k}$ can be taken to be any constant.

(b) $\Delta_{k}^{+}=0$ and $\Delta_{k-m}^{-} \neq 0$ for some $|k| \leq k_{0}$. By (3.2), we get

$$
f_{k}=\frac{1}{\Delta_{k}^{-}}\left(E^{+} g_{k+m}+E^{-} g_{k}\right) .
$$

(c) $\Delta_{k}^{+} \neq 0$ and $\Delta_{k-m}^{-}=0$ for some $|k| \leq k_{0}$. By (3.2), we get

$$
f_{k}=\frac{1}{\Delta_{k}^{+}}\left(E^{+} g_{k}+E^{-} g_{k-m}\right) .
$$

(d) $\Delta_{k}^{+} \neq 0$ and $\Delta_{k-m}^{-} \neq 0$ for some $|k| \leq k_{0}$.

In this situation, $f_{k}$ can be determined as in the proof of Case (i).

In the following, we give the proof of Theorem 1.1.

Proof of Theorem 1.1 From the above discussion, we only need to prove that the function $f(\theta)$ obtained by (1.9) belongs to $L^{2}[-\pi, \pi]$. Obviously, Eqs. (1.1), (1.6) and (3.1) are equivalent to each other. Since $\left\{A_{k}\right\},\left\{B_{k}\right\},\left\{C_{k}\right\},\left\{g_{k}\right\} \in l^{2}$, then $\left\{\Delta_{k}^{ \pm}\right\} \in l^{2}$ and $\Delta_{k}^{ \pm} \neq 0$. It follows 
from (3.3) that $\left\{f_{k}\right\} \in l^{2}$ is a bounded sequence and $\sum_{k=-\infty}^{\infty} f_{k} e^{i k \theta}$ is convergent. Thus, by Lemma 2.2, Eq. (1.1) has a unique solution $f(\theta)=\mathbb{L} f$, and $f(\theta) \in L^{2}[-\pi, \pi]$.

Finally, in order to illustrate that Eq. (1.1) has a solution, we shall present an example. For example, suppose that

$$
\begin{aligned}
& a_{1}=b_{1}=c_{1}=a_{2}=b_{2}=c_{2}=1, \quad a=d=0, \quad b=c, \\
& K_{1}(\theta)=K_{2}(\theta)=\sin \theta, \quad M_{1}(\theta)=M_{2}(\theta)=0, \quad G(\theta)=\sin \theta-\cos \theta,
\end{aligned}
$$

then $\xi(\theta)=\tan \frac{m}{2} \theta$, and Eq. (1.1) can be transformed into

$$
\begin{aligned}
f(\theta) & +\frac{1}{\pi} \int_{-\pi}^{\pi} f(t) \cot \frac{t-\theta}{2} d t+\frac{1}{\sqrt{2 \pi}} \int_{-\pi}^{\pi} \sin (t-\theta) f(t) d t \\
= & \sin \theta-\cos \theta, \quad \theta \in[-\pi, \pi] .
\end{aligned}
$$

Equation (3.17) is often used in engineering mechanics. It is easy to verify that Eq. (3.17) satisfies the above conditions (Assumptions A-C). Via using the methods of Section 3, we can obtain the exact solution of Eq. (3.17):

$$
f(\theta)=\frac{\sqrt{2}}{2}\left(\frac{\pi-\theta}{\pi+\theta}\right)^{\frac{1}{4}} \sin \theta, \quad \theta \in[-\pi, \pi] .
$$

As for the solving method of (3.17), we will not elaborate. We can verify that (3.18) is indeed the solution of (3.17). Therefore, we conclude that a solution set of (1.1) is non-empty.

\section{Homogenous equation and some specific equation}

In this section we consider the homogenous equation and some specific equation. First we consider the homogenous equation (that is, $G(\theta) \equiv 0$ )

$$
a_{1} f+b_{1} H f+K_{1} * f+M_{1} * H f+\xi\left(a_{2} f+b_{2} H f+K_{2} * f+M_{2} * H f\right)=0 .
$$

Via the Laurent transform, it can be reduced to the equation

$$
\Delta_{k}^{+} f_{k}+\Delta_{k-m}^{-} f_{k-m}=0
$$

That is,

$$
f_{k}=-w_{k} f_{k-m}, \quad w_{k}=\frac{\Delta_{k-m}^{-}}{\Delta_{k}^{+}} .
$$

Again we apply the same approach as the discussion for Eq. (3.8) to deduce that $f_{k} \equiv 0$ for all $k$ so that $f(\theta) \equiv 0$. As a result, the homogeneous equation (4.1) has only a trivial solution.

Next we consider the specific case that $\xi(\theta)$ is a constant. Since $a d-b c=0$, Eq. (1.1) can be expressed in the form

$$
a^{\prime} f+b^{\prime} H f+K * f+M * H f=G \text {. }
$$


Via transform $\mathbb{L}^{-1},(4.4)$ can be written as

$$
\left(a^{\prime}+2 i b^{\prime} \operatorname{sgn} k+B_{k}+2 i \operatorname{sgn} k C_{k}\right) f_{k}=g_{k}
$$

where $a^{\prime}, b^{\prime}$ are constants and $\left\{f_{k}\right\}_{k \in \mathbb{Z}}=\mathbb{L}^{-1} f,\left\{B_{k}\right\}_{k \in \mathbb{Z}}=\mathbb{L}^{-1} K,\left\{C_{k}\right\}_{k \in \mathbb{Z}}=\mathbb{L}^{-1} M,\left\{g_{k}\right\}_{k \in \mathbb{Z}}=$ $\mathbb{L}^{-1} G$.

One can solve out $f_{k}$ from (4.5) and get the solution $f(\theta)=\sum_{k=-\infty}^{+\infty} f_{k} e^{i k \theta}$.

\section{Conclusions}

In this paper, we first proposed one class of singular integral equations of convolution type with Hilbert kernel and periodicity. Applying the discrete Laurent transform and its properties, such an equation can be changed into a discrete boundary value problem depending on some parameter, here we call it 'a discrete jump problem'. In this article, our method is different from the ones of the classical boundary value problem, and it is novel and simple. The exact solution, denoted by series, of Eq. (1.1) and the conditions of solvability are obtained. We remark that our approach is also effective to some other classes of equations such as the equations of dual type with periodicity and Hilbert kernel, the Wiener-Hopf type equations, and the equations with periodicity and cosecant kernel. Thus, this paper generalizes the classical theory of boundary value problems and singular integral equations.

One can also consider a similar problem in the setting of Clifford analysis (see, e.g., [1721]).

Acknowledgements
The author is grateful to the referees for many suggestions to improve the exposition of the paper.

Competing interests

The author declares no conflicts of interest.

Authors' contributions

Author read and approved the final manuscript.

\section{Publisher's Note}

Springer Nature remains neutral with regard to jurisdictional claims in published maps and institutional affiliations.

Received: 20 July 2017 Accepted: 27 October 2017 Published online: 09 November 2017

References

1. Giang, BT, Tuan, NM: Generalized convolutions and the integral equations of the convolution type. Complex Var. Elliptic Equ. 55(4), 331-345 (2010)

2. Gomez, C, Prado, H, Trofimchuk, S: Separation dichotomy and wavefronts for a nonlinear convolution equation. J. Math. Anal. Appl. 420, 1-19 (2014)

3. Mohankumar, N, Natarajan, A: On the numerical solution of Cauchy singular integral equations in neutron transport. Ann. Nucl. Energy 35, 1800-1804 (2008)

4. Nakazi, T, Yamamoto, T: Normal singular integral operators with Cauchy kernel. Integral Equ. Oper. Theory 78, 233-248 (2014)

5. Wójcik, P, Sheshko, MA, Sheshko, SM: Application of Faber polynomials to the approximate solution of singular integral equations with the Cauchy kernel. Differ. Equ. 49(2), 198-209 (2013)

6. Noor, MA, Mohyud-Din, ST: Variational iteration technique for solving higher order boundary value problems. Appl. Math. Comput. 189, 1929-1942 (2007)

7. Noor, MA, Noor, KI, Mohyud-Din, ST, Shabbir, A: An iterative method with cubic convergence for nonlinear equations. Appl. Math. Comput. 183, 1249-1255 (2006)

8. Litvinchuc, GS: Solvability Theory of Boundary Value Problems and Singular Integral Equations with Shift. Kluwer Academic, London (2004)

9. Li, PR: Two classes of linear equations of discrete convolution type with harmonic singular operators. Complex Var. Elliptic Equ. 61(1), 67-75 (2016)

10. Li, PR, Ren, GB: Some classes of equations of discrete type with harmonic singular operator and convolution. Appl. Math. Comput. 284, 185-194 (2016) 
11. De-Bonis, MC, Laurita, C: Numerical solution of systems of Cauchy singular integral equations with constant coefficients. Appl. Math. Comput. 219, 1391-1410 (2012)

12. $\mathrm{Du}, \mathrm{H}$, Shen, JH: Reproducing kernel method of solving singular integral equation with cosecant kernel. J. Math. Anal. Appl. 348(1), 308-314 (2008)

13. Jiang, $,, X u, Y$ : Fast Fourier-Galerkin methods for solving singular boundary integral equations: numerical integration and precondition. J. Comput. Appl. Math. 234, 2792-2807 (2010)

14. Li, PR: One class of generalized boundary value problem for analytic functions. Bound. Value Probl. 2015, Article ID 40 (2015). doi:10.1186/s13661-015-0301-0

15. Li, PR: Generalized convolution-type singular integral equations. Appl. Math. Comput. 311, 314-323 (2017)

16. Muskhelishvilli, Nl: Singular Integral Equations. Nauka, Moscow (2002)

17. Gong, YF, Leong, LT, Qiao, T: Two integral operators in Clifford analysis. J. Math. Anal. Appl. 354, 435-444 (2009)

18. Ren, GB, Che, QH, Cerejeiras, P, Kahler, U: Chirp transforms and Chirp series. J. Math. Anal. Appl. 373, 356-369 (2011)

19. Abreu-Blaya, R, Bory-Reyes, J, Brackx, F, De Schepper, H, Sommen, F: Boundary value problems for the quaternionic Hermitian analysis in $\mathbb{R}^{4}$. Bound. Value Probl. 2012, Article ID 74 (2012). doi:10.1687/1687-2770-2012-74

20. Lu, JK: Boundary Value Problems for Analytic Functions. World Scientific, Singapore (2004)

21. Li, PR: Singular integral equations of convolution type with cosecant kernels and periodic coefficients. Math. Probl. Eng. 2017, Article ID 6148393 (2017). doi:10.1155/2017/6148393

\section{Submit your manuscript to a SpringerOpen ${ }^{\circ}$ journal and benefit from:}

- Convenient online submission

- Rigorous peer review

- Open access: articles freely available online

- High visibility within the field

- Retaining the copyright to your article

Submit your next manuscript at $\gg$ springeropen.com 